\title{
Thick to Thin: The Evolutionary Connection Between PG 1159 Stars and the Thin Helium-Enveloped Pulsating White Dwarf GD 358
}

\author{
Benjamin T. Dehner ${ }^{1}$ and Steven D. Kawaler ${ }^{1}$
}

Received __; accepted

\footnotetext{
${ }^{1}$ Dept. of Physics and Astronomy, Iowa State University, Ames IA, 50011; email: btd@iastate.edu
} 


\begin{abstract}
Seismological observations with the Whole Earth Telescope (WET) allow the determination of the subsurface compositional structure of white dwarf stars. The hot DO PG 1159-035 has a helium surface layer with a mass of $\sim 10^{-3} M_{\odot}$, while the cooler DB white dwarf GD 358 has a much thinner surface helium layer of $10^{-6} M_{\odot}$. Taken literally, these results imply that either there is no evolutionary relation between these two stars, or that there is an unknown mass loss mechanism. In order to investigate a possible evolutionary link between these objects, we computed evolutionary sequences of white dwarf models that included time-dependent diffusive processes. We used an initial model based on the PG 1159 pulsational data, which has a surface layer $3 \times 10^{-3} M_{\odot}$ thick, and a composition of $30 \%$ helium, $35 \%$ carbon, and $35 \%$ oxygen. Below this surface layer is a thin transition zone where the helium fraction falls to zero. As expected, diffusion caused a separation of the elements; a thickening surface layer of nearly pure helium overlays a deepening transition zone where the composition changes to the surface composition of the original model. When the model reached the temperature range inhabited by GD 358 and the pulsating DB white dwarfs, this pure helium surface layer was $\sim 10^{-5.5} M_{*}$ deep. The resulting evolved model is very similar to the model used by Bradley and Winget (1994) to match the pulsation observations of GD 358. The pulsation periods of this model also show a good fit to the WET observations. These results demonstrate the plausibility of a direct evolutionary path from PG 1159 stars to the much cooler DB white dwarfs by inclusion of time-dependent diffusion. A problem still remains in that our models have no hydrogen, and thus must retain their DB nature while evolving through the $\mathrm{T}_{\text {eff }} 45,000 \mathrm{~K}$ to $30,000 \mathrm{~K}$. Since there are no known DB stars in this range, we plan to address this problem in future calculations.
\end{abstract}

Subject headings: diffusion — stars: evolution — stars: individual (GD 358, PG 1159) — white dwarfs 


\section{Introduction: The Problem of Ancestry}

Seismological exploration of white dwarf stars with the Whole Earth Telescope, described by Nather et al. (1990), has yielded unprecedented details about their subsurface compositional stratification. Winget et al. (1991) observed the pulsations of the hot $\left(T_{\text {eff }}=140,000 \mathrm{~K}\right)$ white dwarf PG 1159-035 (hereafter simply "PG 1159"), uncovering over 120 independent pulsation modes. These modes appear at frequencies that are naturally explained by pulsation theory as nonradial $g$-modes. Kawaler \& Bradley (1994: "KB") examined the observed pulsation periods in detail, and determined several parameters of this star such as its mass and luminosity. In addition, they show that PG 1159 has a subsurface composition transition zone at about $3 \times 10^{-3} M_{\odot}$ below the surface. The surface composition of that star is roughly $33 \% \mathrm{He}, 50 \%{ }^{12} C$, and $17 \%{ }^{16} O$ by mass, (Werner et al. 1991); the composition transition is where the helium mass fraction goes down to zero. Thus the surface layer contains roughly $10^{-3} M_{\odot}$ of helium. A likely route of evolution to the PG 1159 phase begins with departure from the AGB during a thermal pulse (Iben \& Tutukov 1984). Such a model has at most $10^{-2} M_{\odot}$ of helium in the surface layers. With modest mass loss between the AGB and the PG 1159 stage, the helium layer mass determined by KB fits this picture.

Winget et al. (1994) report observations of the pulsating white dwarf, GD 358. This star is much cooler than PG 1159, and is the prototype of the DB pulsators, with an effective temperature of about $25,000 \mathrm{~K}$ (Thejl, Vennes, and Shipman 1991). Winget et al. (1994) found over 180 separate pulsation frequencies in this star. Model analysis of this star by Bradley \& Winget (1994: "BW") successfully reproduced in detail the pulsation frequencies with a model of about the same mass as PG 1159, but with a surface layer of pure helium of approximately $1.2 \times 10^{-6} M_{\odot}$. This is three order of magnitude smaller than PG 1159 .

This difference in helium layer mass suggests that establishing an evolutionary connection between these two objects requires finely-tuned mass loss beyond the PG 1159 stage. Alternatively, these results challenge the notion of there being a direct evolutionary relationship between these objects.

The models used by KB were evolutionary models which had a single common ancestor on the AGB. The time that they took to reach the PG 1159 stage was less than $10^{6}$ yr. The models used by BW were also evolutionary models with ages of several $\times 10^{7}$ years after departure from the AGB. In both calculations, the compositional stratification was fixed at the start, and did not change in the course of the calculation. However, diffusion by gravitational settling, acting on reasonably short time scales, is believed to be responsible for the extremely pure surface compositions of most white dwarfs stars (Schatzman 1958). While there has been much discussion of diffusion in the literature, there have few attempts to include time-dependent diffusion as part of an evolutionary calculations (see for example Iben and MacDonald 1985). Other white dwarf evolution calculations that address diffusion usually assume that diffusion reaches equilibrium quickly, and so evolve models with equilibrium diffusion profiles in their interiors (e.g. Tassoul, Fontaine, \& Winget 1990). The time scale to reach equilibrium increases dramatically with depth and with a shallow gradient. In realistic models, diffusion takes a long time to approach equilibrium in deeper layers, and equilibrium concentrations are reached from the outside inward. Therefore, the use of equilibrium (or near-equilibrium) diffusive profiles is open to question in objects as young as PG 1159 and GD 358. BW's evolutionary models employ an equilibrium diffusion profile above the midpoint of the composition transition region, and enforce a steeper gradient below, which crudely models the approach to diffusive equilibrium in deeper layers.

This paper reports on results of our calculations of time-dependent diffusion in evolving white dwarf models. Since diffusive equilibrium is only truly achieved after an infinite time, we constructed a series of 
evolutionary models including time-dependent diffusive processes. We find that GD 358 is a "snapshot" of diffusion in progress; it represents an intermediate step in the approach to diffusive equilibrium. Its outer layer structure is a natural consequence of the evolution of the compositional structure of a PG 1159-like star. In Section II below we briefly describe the implementation of diffusion within our white dwarf evolution code. In Section III, we show the results of evolution of models with starting models taken from $\mathrm{KB}$, and compare the results to the models used by BW. Since pulsation periods and their differences are the prime observational test of the model, in Section IV we compare the pulsation properties of our models with GD 358. Section V concludes this paper with a discussion of the evolutionary link between the PG 1159-035 stars and the DB stars.

\section{Evolutionary Models of White Dwarfs with Time-Dependent Diffusion}

The computer code we used was a standard stellar evolution code (ISUEVO; Dehner, 1995) which was optimized for use in pulsation studies. This code was modified to include time-dependent diffusion in the following way. The equation describing diffusion is

$$
\frac{\partial n_{i}}{\partial t}=-\frac{1}{r^{2}} \frac{\partial\left(r^{2} w_{i} n_{i}\right)}{\partial r}
$$

where $n_{i}$ is the number density of species $i$ (element or electrons), $t$ is time, $w$ is the diffusive velocity, and $r$ the radial coordinate. The code solves equation [1] using a technique known as the method of lines. First, equation [1] is spatially discretized using centered differences. Each evolutionary time step is then broken down into substeps. Within each substep, the set of discretized diffusion equations is integrated for each element using the backward differentiation integrator DEBDF by Shampine and Watts (1979), after which the abundances are updated. Typically, the first few substeps are chosen to be very short (5 years), with subsequent steps being determined by considering the maximum composition change at all zones from the previous step.

The diffusive velocity $w_{i}$ is obtained using the following equation derived from Burgers (1969)

$$
\nabla p_{i}-\frac{\rho_{i}}{\rho} \nabla p-n_{i} Z_{i} e \mathbf{E}=\sum_{j}^{M+1} K_{i j}\left(\mathbf{w}_{j}-\mathbf{w}_{i}\right)
$$

where $i$ and $j$ are species indices, $Z$ is the electric charge, $\mathbf{E}$ is the electric field, and $K_{i j}$ is the resistance coefficient, and all other symbols have their usual meanings. The resistance coefficients, inversely related to the diffusion coefficients used by some authors, are obtained from the tables of Paquette et al. (1986). Equation [2] follows from Burgers (1969) after assuming that thermal diffusion and magnetic field effects are negligible (cf. Burgers 1969, eqn. [18.1].)

We also ignore the effect of radiative forces. The principal aim of this paper is to explore time-dependent diffusion of dominant species. Radiative forces might result in modest increases in the abundances of heavy elements, but probably would not significantly modify the helium abundance in terms of the pulsation diagnostics. We recognize that radiative levitation studies are very fashionable in the white dwarf literature because of the importance of heavy element effects on white dwarf fluxes. Clearly, radiative forces are important for hot white dwarfs such as those detected by EUVE, ROSAT, et al.; we are currently working on including simple radiative levitation to address other issues in white dwarf evolution. 
We evolved four evolutionary sequences with masses from $0.56 M_{\odot}$ to $0.62 M_{\odot}$, to bracket the mass determined by BW for GD 358. The starting model for these sequences was the $0.59 M_{\odot}$ static model which best reproduced the pulsation spectrum of PG 1159 from KB. This model, with $T_{\text {eff }}=140,000 K$, had a surface layers with uniform composition of $30 \%$ helium, $35 \%$ carbon, and $35 \%$ oxygen. In a transition region at $3 \times 10^{-3} M_{*}$ below the surface, the composition changed to $\mathrm{C}=\mathrm{O}=50 \%$. The thermal structure of this model is that of an evolutionary model of a post-AGB star which left the AGB during a helium shell flash. To obtain starting models at different masses, we simply re-integrated the model with the chosen mass so as to reach a consistent central boundary condition. This procedure results in realistic models that closely match evolutionary models with similar masses and histories. For a comparison between these starting models and full evolutionary models, see KB. Evolution proceeded into the temperature domain of the DB variables; when convection developed in these models we computed the convective flux using the ML2 formulation of Tassoul, Fontaine, \& Winget (1990).

\section{Model Results: The Evolutionary Connection}

The results of the diffusion calculations reveal, as expected, that carbon and oxygen sink downward, and helium floats to the top. Figure 1 shows the helium mass fraction as a function of the stellar mass fraction below the surface $\left(q_{s}\right)$ for several models in the $0.58 M_{\odot}$ sequence. Models in the other sequences evolve in very similar ways. The starting model shows a constant helium abundance down to $\log q=-3.1$; the helium abundance drops to zero at $\log q=-2.6$. After 400,000 years, the model reaches an effective temperature of $76,000 \mathrm{~K}$. At this point, a steep composition gradient has developed at $\log q=-8.0$. Below this gradient, the abundance drops gradually down to the original surface helium abundance of $30 \%$. The initial composition transition at $\log q=-2.6$ is essentially unchanged. At $2 \times 10^{6}$ yr after the start $\left(T_{\text {eff }}=46,100 K\right)$, the outer composition transition zone lies at $\log q=-7.5$. Once the model reaches the $T_{\text {eff }}$ range where pulsating DB white dwarfs are found (in Figure 1, this is the model at $T_{\text {eff }}=28,600 K$ ) at an age of $8.8 \times 10^{6} \mathrm{yr}$, the outer composition transition has reached down to $\log q=-6.5$. As the model evolves through the instability strip, this $\mathrm{He} / \mathrm{C}$ transition zone deepens further, $\operatorname{reaching} \log q=-6.0$ at $T_{\text {eff }} \sim 24,000 K\left(2.0 \times 10^{7} \mathrm{yr}\right)$, and deepening to $\log q=-5.6$ at $T_{\text {eff }} \sim 21,000 K\left(4.1 \times 10^{7} \mathrm{yr}\right)$. Even in these later models, diffusion has only slightly broadened the zone where the helium abundance drops from the original surface value to zero. This is to be expected because of the very long diffusion time scales that deep in the star.

The models of BW were constructed using an entirely different evolutionary code and input physics, we compare the global parameters of our models with their best model for GD 358 to see how similar the models are. Bradley (private communication) reports that their best model had $M=0.61 M_{\odot}, T_{\text {eff }}=24,210$, $\log \left(L / L_{\odot}=-1.3077\right.$, and $R=0.01275 R_{\odot}$. The closest model in our $0.60 M_{\odot}$ sequence was at $T_{\text {eff }}=24,172 ;$ that model had a luminosity of $\log \left(L / L_{\odot}\right)=-1.3125$ and a radius of $0.01263 R_{\odot}$. Most significantly, the composition transition zone lies at the same position below the surface in both models. Thus, the global parameters for the diffusion models and the models of BW are quite similar. The evolutionary sequence that we calculated produced a model that is extremely close to the model of BW that was designed to fit the observed properties of GD 358.

In our models, the starting point was a tested stellar model that reproduced the observations of a much younger object than GD 358. Therefore, evolution of these stars leads to a natural and direct evolutionary connection between the prototype of the PG 1159 stars and the prototype of the DB pulsators. 


\section{Testing the Connection}

BW used the pulsation periods observed in GD 358 to constrain the compositional structure of the outer layers of the star. The observation that demanded a thin helium layer was the nonuniformity of the spacing between successive overtone periods in that star. Through the effects of mode trapping (KB, Brassard et al. 1992, Bradley, Winget \& Wood 1992), these departures occurred regularly, with the number of modes between minima in the spacing increasing with decreasing surface layer thickness. For GD 358, the fit was to a single pronounced minimum in the period spacing, because of the limited number of certain overtone periods seen by Winget et al. (1994).

To see how well our models match the observed pulsation properties of GD 358, we computed pulsation periods for all models in all four sequences that were in or near the DB instability strip. We then compared the computed periods to those seen in GD 358 by Winget et al. (1994), and looked for those models which most closely matched the star. We used two quantitative comparisons: the periods themselves, and the differences between successive periods. The mean differences between the observed and computed periods (and the period spacings) reaches a minimum for the models that are the closest fits to GD 358. It should be stressed that the periods and the period spacings are two separate criteria for comparing models with observations.

To compare each model with observations, we computed the sum of the squares of the differences between the periods of each model and GD 358, and did the same for the period spacings. Figure 2 shows the inverse sum-of-squares difference in the periods, and in the period spacings, as a function of effective temperature for the three sequences bracketing the $\mathrm{BW}$ value of $0.58 M_{\odot}$. Remarkably the minimum difference (which appear as peaks in this plot) for both the periods and the period spacings are very close together for these sequences. The overall best model is the $0.58 M_{\odot}$ model at $T_{\text {eff }}=24,121$. The mean period spacing of this model is $39.52 \pm 3.2 s$, compared to the observed value of $39.5 \pm 5.2$. In comparison with the models of $\mathrm{BW}$, while they have an overall better fit to the observed pulsational properties of GD 358, our models have the advantage of not being tuned to fit the observations, but were evolved directly from the PG 1159 stage.

\section{Conclusions: An Evolutionary Link Between DBV Stars and PG 1159 Stars}

Our models demonstrate how chemical diffusion can cause PG 1159 stars to evolve into DBV stars such as GD 358 without appeal to mass loss, despite the incongruity in surface helium layer masses. This gives one definitive path of evolution of white dwarf stars from the PG 1159 stage, and is a step in clarifying white dwarf evolutionary relationships. This also demonstrates the necessity of invoking time-dependent diffusion when considering white dwarf evolution.

In this investigation, we assumed a zero hydrogen fraction in all of our models. This implies that that they must retain their DB nature while cooling through the DB gap from $45,000 \mathrm{~K}$ to $30,000 \mathrm{~K}$. This is an obvious contradiction with the observed lack of DB stars in this temperature range (Wesemael et al. 1985; Liebert 1986). In future calculations with trace hydrogen present, we will examine the behavior of the hydrogen and helium layers in this range. 
This work was supported by NSF Grant AST-9115213 and NSF Young Investigator Award AST9257049 to Iowa State University. We also thank Ed Nather for his continual insistence on quantifying the comparison of models with the observations. 


\section{REFERENCES}

Bradley, P. \& Winget,D. 1994, ApJ, 430, 850 (BW)

Bradley, P., Winget, D., \& Wood, M., 1992, ApJ, 406, 661

Brassard, P., Fontaine, G., Wesemael, F., \& Hansen, C.J., 1992 ApJS, 80, 369

Burgers, J., Flow Equations for Composite Gases, 1969, (New York and London: Academic Press)

Dehner, B.T., 1995, Ph.D. thesis, Iowa State University

Iben, I., Jr., \& MacDonald, J., 1985, ApJ, 296, 540

Iben, I., Jr., \& Tutukov, A.V., 1984, ApJ, 282, 615

Kawaler, S.D., 1990, Confrontation Between Stellar Pulsations and Evolution, ed. C. Cacciari \& G. Clementini, (San Fransico: ASP)

Kawaler, S.D., \& Bradley, P., 1994, ApJ, 427, 415 (KB)

Liebert, J., 1986, Hydrogen Deficient Stars and Related Objects, eds. K. Hunber, D. Schönberner \& N.K. Rao (Dordrecht: D. Reidel)

Nather, R.E., Winget, D.E., Clemens, J.C., Hansen, C.J., \& Hine, B.P., 1990, ApJ, 361, 309

Paquette, C., Pelletier C., Fontaine, G., \& Michaud, G., 1986, ApJS, 61, 177

Schatzman, E., 1958, White Dwarfs, (Amsterdam: North-Holland)

Shampine, L.F., \& Watts, H.A., SAND79-2374

Tassoul, M., Fontaine, G., and Winget, D. 1990, ApJS, 72, 335

Thejl, P., Vennes, S., \& Shipman, H.L.,1991, ApJ, 370355

Werner, K., Heber, U., \& Hunger, K. 1991, A\&A, 244, 437

Wesemael, F., Green, R.F. \& Liebert, J., 1985, ApJS, 58, 379

Winget, D.E., et al., 1994, ApJ, 430, 839 


\section{FIGURE CAPTIONS}

Fig. 1 Composition profiles for various models. The $\mathrm{x}$ axis is the $\log$ of the surface mass fraction, given by $q_{s}=\left(M_{*}-M_{r}\right) / M_{\odot}$.

Fig. 2 Inverse sum-of-squares comparison of pulsation periods and period spacings to GD 358 observations for all models in the DB instability strip. 


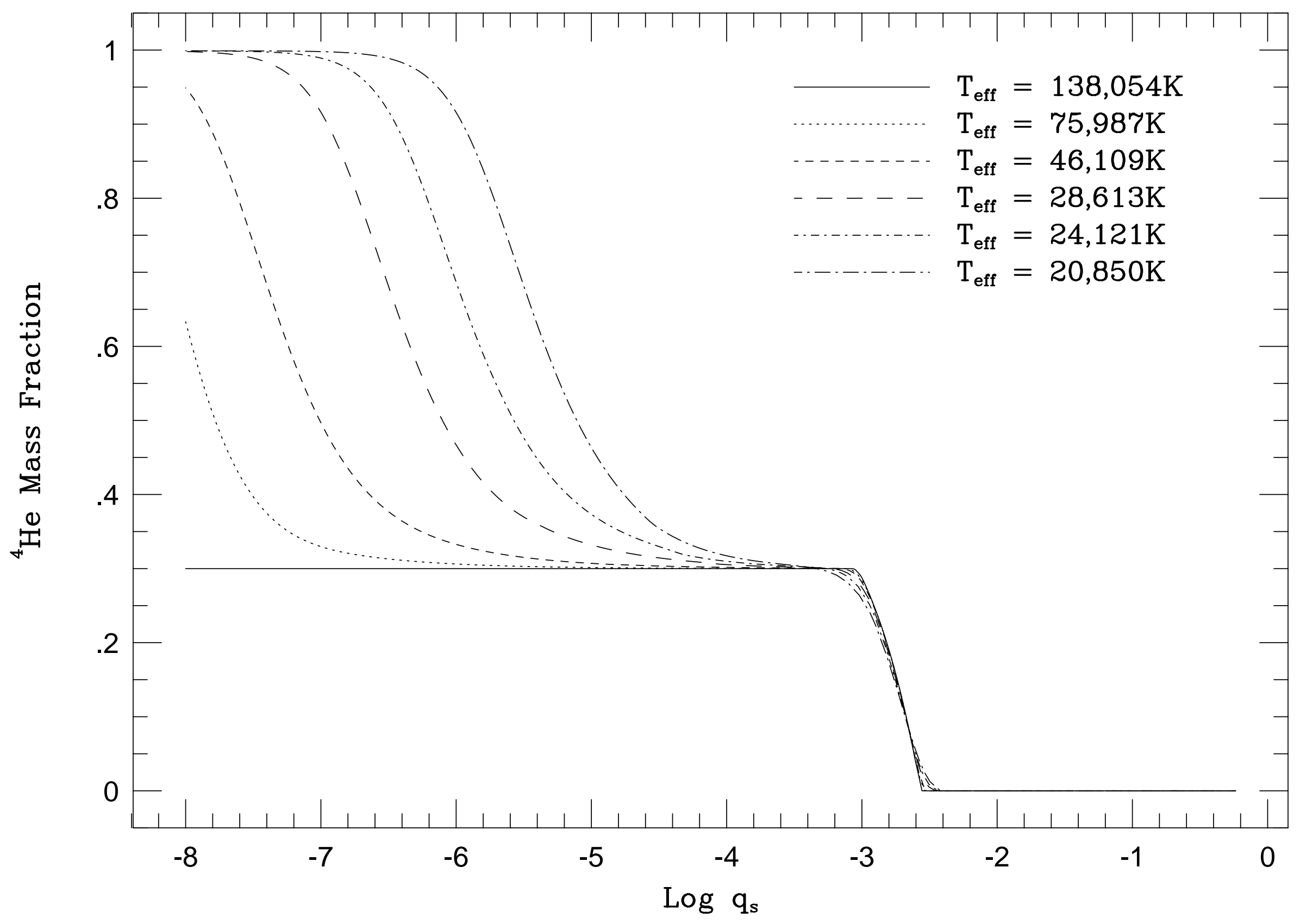




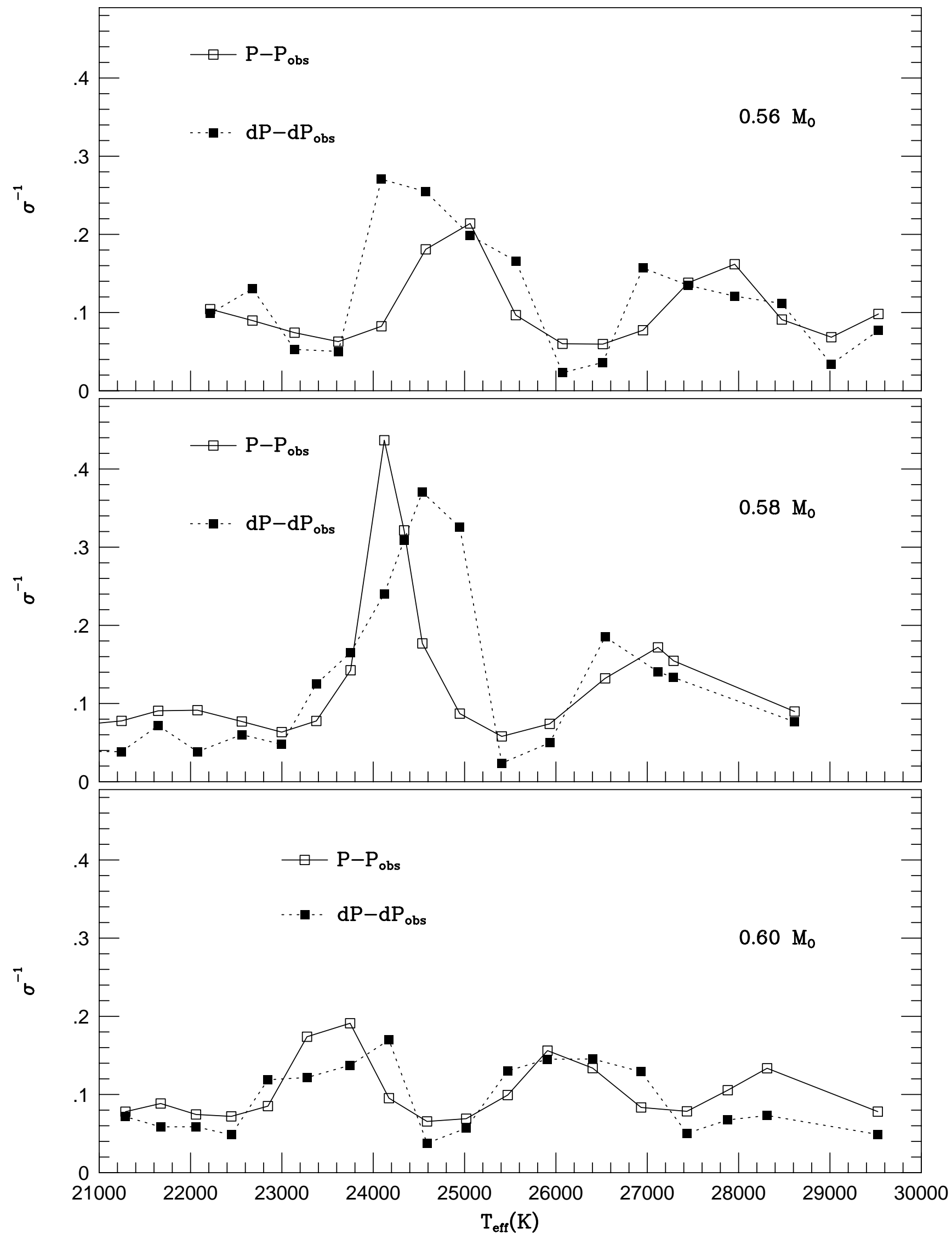

